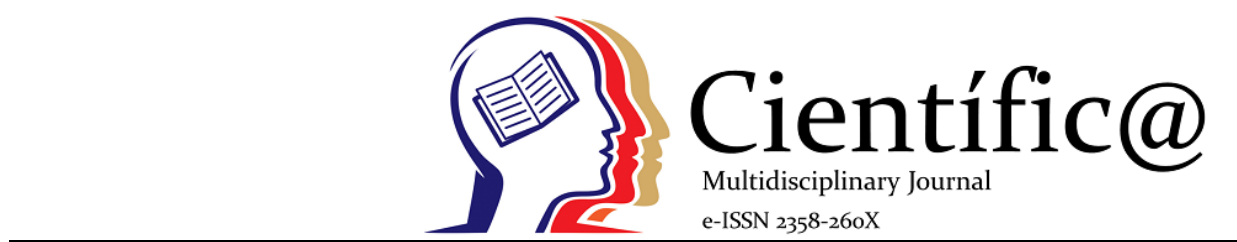

\title{
AVALIAÇÃO DAS CARACTERÍSTICAS FÍSICO-QUÍMICAS, ATIVIDADE ANTIOXIDANTE E FENÓLICOS TOTAIS DA FARINHA DO EXTRATO DA JABUTICABA (Myrciaria jaboticaba).
}

\section{EVALUATION OF PHYSICAL CHEMICAL CHARACTERISTICS, ANTIOXIDANT ACTIVITY AND TOTAL PHENOLICS OF JABUTICABA EXTRACT FLOUR (Myrciaria jaboticaba)}

\author{
Talita Azevedo dos Santos ${ }^{1 *}$; Joel Pimentel de Abreu ${ }^{2}$; Taíssa Lima Torres ${ }^{3}$
}

\author{
${ }^{1}$ Universidade Federal do Estado do Rio de Janeiro, Rio de Janeiro/ Rio de Janeiro/Brasil \\ talitasantosazevedo@gmail.com \\ ${ }^{2}$ Universidade Federal do Estado do Rio de Janeiro, Departamento de Techologia dos Alimentos, Rio de Janeiro/ Rio de \\ Janeiro/Brasil \\ ${ }^{3}$ Universidade Federal do Estado do Rio de Janeiro, Departamento de Nutrição Aplicada, \\ Rio de Janeiro/Rio de Janeiro/Brasil
}

\section{Info}

Recebido: 08/2020

Publicado: $12 / 2020$

DOI: 10.29247/2358-260X.2020v7i2.4730

ISSN: 2358-260X

\section{Palavras-Chave}

Aproveitamento, Jabuticaba, compostos

bioativos, casca e polpa.

\section{Keywords:}

Utilization, Jabuticaba, bioactive

compounds, peel and pulp.

\begin{abstract}
The jabuticaba Myrciaria cauliflora (is a native Brazilian fruit with high antioxidant potential, but with low commercialization and consumption, being a fruit of high perishability and large residue generation. The physicalchemical characterization as well as the analysis of the antioxidant activity of the residue. jabuticaba aims to provide subsidies for the development of by-products with healthy and sustainable added value, which allow greater accessibility to this raw material. In the present study was prepared a flour of jabuticaba extract (using the peel and pulp) preceded by physicochemical analysis and antioxidant activity analysis using the Frap and Abts methods, total phenolics.The jabuticaba extract flour presented a yield of $18 \%$. Humidity $(11.29 \%)$, ashes (3
\end{abstract} Acidity (1.8\%) and $\mathrm{pH}(3.5)$, total sugars (62.43\%), lipids (0.6\%), proteins $(12.13 \%)$ and fiber $(9,35 \%)$ were adequate. Total phenol and dolic acid content was: $1419.8 \mu \mathrm{mol}$ Fe2 + / g (FRAP) and $2505.53 \mu$ mol Trolox / $\mathrm{g}$ (ABTS) and 5410.3mg GAE / 100g. These results are superior to several other fruit flours documented in the literature. Therefore, jabuticaba extract flour is a viable way of making this fruit available in a sustainable way, besides presenting itself as an alternative source of antioxidants, to be used in the elaboration of healthy products.

\section{Resumo}

A jabuticaba Myrciaria cauliflora (é uma fruta nativa brasileira, com alto potencial antioxidante, mas com baixa comercialização e consumo, por ser uma fruta de alta perecibilidade e grande geração de resíduo. A caracterização físico-química bem como a análise da atividade antioxidante do resíduo da jabuticaba objetiva fornecer subsídios, para fins de desenvolvimento de subprodutos com agregação de valor saudável e sustentável, que permitam a maior acessibilidade a esta matéria-prima. No presente estudo foi elaborada uma farinha do extrato da jabuticaba (utilizando a casca e a polpa) que precedeu-se a analises físico- químicas e analise de atividade antioxidante, utilizando os métodos de Frap e Abts; fenólicos totais. A farinha do extrato da jabuticaba apresentou um rendimento de 18\%. Umidade (11,29\%),cinzas (3,5\%),acidez(1,8\%) e pH $(3,5)$, de açúcares totais $(62,43 \%)$, lipídeos $(0,6 \%)$, proteínas $(12,13 \%)$ e fibras $(9,35 \%)$ estavam adequados. A atividade antioxidante e fenólica totais apresentada foi de: 1419,8 $\mu$ mol Fe2+/g (FRAP) e 2505,53 $\mu \mathrm{mol}$ Trolox/g (ABTS) e 5410,3mg GAE/100g. Estes resultados se expressam superiores a várias outras farinhas de frutas documentadas na literatura. Portanto a farinha do extrato da jabuticaba é uma forma viável de disponibilizar este fruto de forma sustentável, além de se apresentar como uma alternativa fonte de antioxidantes, a ser utilizada na elaboração de produtos saudáveis. 


\section{INTRODUÇÃO}

O Brasil ocupa posição de destaque internacional em biodiversidade, devido ao seu clima privilegiado e território fértil disponível. Atualmente é o segundo maior produtor agrícola mundial e terceiro no ranking da produção mundial de frutas (Instituto de Economia Agrícola, 2019). No entanto, existe um contraste entre a produção agrícola brasileira e o desperdício de milhares de produtos que poderiam ser aproveitados no país (DERAL, 2017).

A jabuticaba é uma fruta nativa do Brasil, pertence à família Myrtaceae, sendo as principais espécies representadas pela Myrciaria jaboticaba (Vell.) e O. Berg (Donadio, 2000). Embora a jabuticaba seja uma fruta apreciada em todo território brasileiro, raramente é utilizada comercialmente pela indústria, devido ao elevado investimento econômico em função da pequena sazonalidade e da alta perecibilidade do fruto (Associação Brasileira de Franchising, 2015).

A maior parte do cultivo brasileiro da jabuticaba se faz na região sudeste do país e, em pequena escala pela agricultura familiar, destinada apenas à produção artesanal de suco, doce, geléia, vinagre, licores e vinhos (Danner et al., 2010). Normalmente, na fabricação dos subprodutos da jabuticaba, as cascas e sementes, onde se encontram a maior parte dos compostos bioativos do fruto, são desprezadas, sendo que estas representam quase $50 \%$ do fruto (Oliveira et al., 2003)(Oliveira et al., 2003).

Em algumas regiões do país, a comercialização da jabuticaba é realizada principalmente na forma in natura às margens de rodovias, advindos de agricultura familiar. Dessa forma, a colheita dos frutos nativos pelo sistema extrativista reveste-se de importância econômica e social (Danner et al., 2010).

O fruto de jabuticaba é uma baga, subgloboso, quando maduro, liso, com 1,6 a 2,2 cm de diâmetro, contendo de uma a quatro sementes. A casca apresenta coloração roxa escura e a polpa esbranquiçada, de gosto doce, ligeiramente adstringente e sabor apreciável (Alezandro et al., 2013) .

Apesar de cada variedade e cada parte da jabuticaba, casca, semente e polpa, possuir uma composição ligeiramente diferenciada, seus principais constituintes são: água, carboidratos principalmente glicose e frutose e fibras, além de considerável teor de vitaminas e minerais (Alezandro et al., 2013).

Além dos atributos nutricionais, a jabuticaba apresenta potencial funcional, dado a ampla variedade de compostos fenólicos, tais como antocianinas, que se concentram majoritariamente nas cascas, de elevada atividade antioxidante (Bicudo, Ribani e Beta, 2014; Borges et al., 2011; Kang et al., 2012; Rufino et al., 2010; Wu et al., 2012). Estes compostos bioativos estão associados a potenciais benefícios à saúde humana (Nile e Park, 2014) .

Evidências científicas apontam que dietas baseadas no consumo de frutas e vegetais têm sido relacionadas a benefícios à saúde humana, devido à presença de compostos bioativos (fitoquímicos), presentes principalmente na casca (Halvorsen et al., 2006; Yang et al., 2016) (Halvorsen et al., 2006; Yang et al., 2016). Em recente estudo (Torres e Farah, 2017) identificaram um grande número desses compostos bioativos em alimentos regionais nativos brasileiros, com destaque para a jabuticaba.

O fruto da jabuticaba considerado uma "berry" brasileira apresenta relevância por seu potencial nutricional e funcional, devido a concentrações expressivas de polifenóis, como cianidina, glucósido, delfinidina-3-O-glucósido, ácido gálico, rutina, miricetina e quercetina (Leite-Legatti et al., 2012; SANTOS, VEGGI e MEIRELES, 2010) .

Estudos experimentais e clínicos evidenciam que os benefícios a saúde atribuídos aos polifenóis presentes na jabuticaba estão fortemente associados ao 
seu potencial antioxidante, com ação anti-inflamatória, melhora do perfil lipídico e da sensibilidade a insulina, modulação da microbiota intestinal e seus metabólitos, o controle do apetite, a redução da absorção intestinal e a síntese lipídica no fígado (Alezandro et al., 2013; Leite-Legatti et al., 2012; Silva, Da, 2017; Szajdek e Borowska, 2008; Wu, Long e Kennelly, 2013) , indicando a jabuticaba ser um fruto capaz de ajudar na prevenção e tratamento de doenças crônicas.

Em virtude de seus atributos sensoriais, este fruto possui elevado potencial de mercado (Barros, Finger e Magalhães, 1996). O processamento da jabuticaba pode ser de grande relevância para garantir o seu consumo contínuo, já que os frutos destas espécies apresentam curta vida pós-colheita.(Vizzotto e Pereira, 2009).

A insuficiência de informações científicas quanto à composição nutricional de cada uma das partes comestíveis da jabuticaba, bem como o não aproveitamento integral da mesma, pode contribuir para o aumento do desperdício desses recursos alimentares no país. Por outro lado, a utilização comercial sustentável destas frutas pode ajudar na preservação das áreas plantadas no Brasil (Braga A C D, Lima M S, Azevedo L C, 2011).

Sob o ponto de vista da sustentabilidade, a elaboração de subprodutos a partir dos resíduos da jabuticaba, pode ser considerada uma alternativa de melhor aproveitamento dessas, favorecendo o desenvolvimento social, econômico e ambiental do Brasil.

A caracterização físico-química bem como a análise da atividade antioxidante do resíduo da jabuticaba objetiva fornecer subsídios, para elaboração de produtos a partir deste.

\section{MATERIAL E MÉTODOS}

\section{Obtenção da amostra}

As jabuticabas foram adquiridas do Centro de Abastecimento da Cidade do Rio de Janeiro (CADEG) no mês de novembro de 2014, respeitando a sazonalidade do fruto.

A farinha do extrato da jabuticaba foi obtida por secagem do resíduo (polpa e casca) resultante da formulação de suco integral de jabuticaba, desenvolvido por projeto de pesquisa de frutos nativos do Brasil (PIBIC/UNIRIO). O resíduo foi extraído na finalização do processo de fabricação do suco integral da jabuticaba, por esmagamento e esgotamento final, oriundos da casca e semente da jabuticaba. A partir dos dados de pesagem inicial dos frutos para elaboração do suco integral, os resíduos (polpa, casca e semente) foram pesados em balança analítica. A secagem foi realizada em estufa de recirculação de ar à $65^{\circ} \mathrm{C}$ por $16-$ 20 horas . Após a desidratação foi realizada a separação da polpa, casca e sementes da jabuticaba, posteriormente as sementes foram descartadas, sendo o bagaço (polpa e casca) desidratado e processado em moinho analítico na granulometria a 56 mesh (moinho analítico).

\section{Caracterização Físico-Química}

\section{Determinação do pH}

$\mathrm{O}$ pH da farinha do resíduo da jabuticaba foi determinado em medidor de $\mathrm{pH}$, modelo portátil DM pH-2, Hanna Instruments, com inserção do eletrodo diretamente na solução a 10\% de farinha do extrato da jabuticaba em água destilada (IAL, 2008). Feito em triplicada.

\section{Umidade}

O teor de umidade da farinha do extrato da jabuticaba foi obtido por método gravimétrico, calculando a perda de peso em estufaa $105^{\circ} \mathrm{C}$, até pesagem constante (IAL, 2008). Feito em triplicada. 


\section{Resíduo Mineral Fixo (RMF)}

O teor de cinzas da farinha do extrato da jabuticaba foi determinado por método gravimétrico com anterior incineração das amostras a $550^{\circ} \mathrm{C}$ segundo o método (IAL, 2008). Feito em triplicada.

\section{Extração de lipídeos}

A extração contínuacom éter etílico em aparelho do tipo Soxhlet foi usada na determinação do extrato etéreo(IAL, 2008). Feito em triplicada.

\section{Análise de proteínas}

$\mathrm{Na}$ análiseda fração protéica utilizou-se o método Kjeldahl modificado utilizando-se 6,25 como fator de conversão (IAL, 2008). Feito em triplicada.

\section{Determinação de fibras}

A fibra bruta foi determinada por gravimetriapela digestão em meio ácido, seguida por uma digestão em meio alcalino(IAL, 2008) . Feito em triplicada.

\section{Açúcares Totais}

Os açúcares totais da farinha do extrato da jabuticaba foram calculados a partir da subtração das percentagens acima de 100\%. Feito em triplicada.

\section{Acidez Total Titulável}

A acidez total titulável (ATT) foi medida por titulação do filtrado da solução com $10 \%$ de farinha do extrato da jabuticaba, com $\mathrm{NaOH} 0,1 \mathrm{M}$ padronizado segundo técnica estabelecida pelas normas do Instituto Adolfo Lutz (2008) e os resultados expressos em \% de acidez total. Feito em triplicada.

\section{Ensaios de Atividade Antioxidante in vitro}

\section{Reagentes}

2,2-Azino-bis (3-ethylbenzo-thiazoline-6sulfonic acid) diammoniumsalt (ABTS), persulfato de potássio, 6-hydroxy-2,5,7,8-tetramethylchroman-2carboxylic acid (Trolox $\left.{ }^{\circledR}\right)$ e 2,4,6-tripyridyl-S-triazine (TPTZ) foram adquiridos da Sigma-Aldrich (Alemanha). Folin-Ciocalteureagent. Sulfato ferroso heptahidratado e hexacianoferrato (II) de potássio foram adquiridos da Merck (Alemanha). Cloreto férrico hexahidratado da Vetec Química Fina (Brasil) e etanol absoluto da Tedia Brazil (Brasil). Metanol, acetona, Água Milli-Q (Millipore, Brasil) foi utilizada em todos os experimentos.

Extração dos compostos antioxidantes e dos compostos fenólicos

A extração da farinha do extrato da jabuticaba foi realizada com a solução extratora sequencial de metanol $50 \%$ e acetona $70 \%$ (Rufino et al., 2009) . Foi utilizado banho de ultrassom por 30 minutos e após o extrato foi avolumado com água destilada. Feito em triplicada.

\section{Extração da Atividade antioxidante}

A atividade antioxidante da farinha do resíduo da jabuticaba foi avaliada usando dois diferentes ensaios in vitro: FerricReducingAntioxidant Power - FRAP (Benzie e Strain, 1999) e TroloxEquivalenceAntioxidantCapacity - ABTS (RE et al., 1999), adaptadas por (Rufino et al., 2010). Feito em triplicada.

\section{Ensaio TEAC}

O radical ABTS $\bullet+$ foi produzido reagindo-se 7 $\mathrm{mM}$ de ABTS com 2,45 mM de persulfato de potássio em água por 12 a 16 horas a temperatura ambiente e ao abrigo de luz. A solução aquosa de ABTS•+ foi diluída com etanol PA (1:100), de maneira a obter-se uma solução com absorbância de $0,70 \pm 0,02$ a $734 \mathrm{~nm}$. 
Alíquotas de amostra foram adicionadas a $2,5 \mu \mathrm{L}$ da solução de ABTS•+ e etanol para completar o volume de 3mL. A absorbância a $734 \mathrm{~nm}$ foi medida após a incubação por 6 min a $37^{\circ} \mathrm{C}$, sendo esse valor utilizado no cálculo da variação de absorbância. Uma curva de calibração foi construída a partir da variação da absorbância ocasionada por concentrações crescentes de trolox, sendo o resultado final de TEAC expresso em $\mu$ molTrolox por grama da farinha do extrato de jabuticaba. Feito em triplicada.

\section{Ensaio FRAP}

Alíquotas de 2,7 $\mu \mathrm{L}$ da amostra foram adicionada a $270 \mathrm{~mL}$ de água $+90 \mu \mathrm{Lda}$ solução reagente de FRAP, preparada no dia da análise. A absorbância a $595 \mathrm{~nm}$ foi medida após a incubação por 30 minutos a $37^{\circ} \mathrm{C}$. Os valores de FRAP foram obtidos comparando-se a modificação de absorbância na mistura teste com aquelas obtidas a partir de concentrações crescentes de $\mathrm{Fe}^{2+}$. $\mathrm{O}$ resultado foi expresso em $\mu$ molde Sulfato ferroso por grama da farinha do extrato de jabuticaba. Feito em triplicada.

\section{Quantificação dos compostos fenólicos}

O teor de compostos fenólicos totais foi determinado pelo método espectrofotométrico com reagente de Folin- Ciocalteau segundo metodologia adaptada proposta por (Singleton, Orthofer e LamuelaRaventós, 1999). Alíquotas da amostra foram adicionados a $2.5 \mathrm{~mL}$ de folin e a $2 \mathrm{~mL}$ de carbonato de cálcio; abrigadas da luz por duashoras. Foi utilizado ácido gálico como padrão de referência. Este método envolve a redução do reagente pelos compostos fenólicos das amostras com formação de um complexo azul.

A absorbância a $750 \mathrm{~nm}$ foi mensurada e os valores de fenólicos totais nos extratos expressos em mg GAE/100 de amostra.

Os espectros de absorção do extrato da farinha do resíduo de jabuticaba, na região entre 593 e 750 nm, foram obtidos em espectrofotômetro UV-1800 (Shimadzu, Japão). Feito em triplicada.

\section{Análise Estatística}

Os dados resultantes da avaliação da atividade antioxidante in vitro (FRAP e ABTS), fenólicos totais (Folin-Ciocalteu), umidade, rendimento, cinza, pH, acidez total e açúcares totais são apresentados como médias \pm Desvio Padrão, por triplicata de leitura de cada amostra no programa de Análise de variância (ANOVA) .

\section{RESULTADOS E DISCUSSÃO}

A farinha do resíduo da jabuticaba apresentou um rendimento de $17 \%$, em relação o resíduo que apresenta- se similar ao obtido por (Mendes, 2013), em farinha da casca da manga, que apresentou rendimento de $19,8 \%$.

A caracterização físico-química da farinha do resíduo da jabuticaba é apresentada na Tabela 1.

Tabela 1. Caracterização físico-química da farinha do extrato da jabuticaba ${ }^{1}$.

\begin{tabular}{lcccccccc}
\hline & $\begin{array}{c}\text { Umidade } \\
\mathbf{( \% )}\end{array}$ & $\mathbf{p H}$ & $\begin{array}{c}\text { Acidez } \\
\text { total } \mathbf{( \% )}\end{array}$ & $\begin{array}{c}\text { Cinzas } \\
\mathbf{( \% )}\end{array}$ & $\begin{array}{c}\text { Açúcares } \\
\text { totais }\end{array}$ & Lipídeos & Proteínas & Fibras \\
\hline $\begin{array}{l}\text { Jabuticaba } \\
\text { (farinha do }\end{array}$ & $11,29 \pm 0,12$ & $3,5 \pm 0,3$ & $1,8 \pm 0,4$ & $3,5 \pm 0,2$ & $62,43 \pm 1,19$ & $0,6 \pm 0,06$ & $12,83 \pm 0$ & $9,35 \pm 1,26$ \\
extrato) & & & & & & & &
\end{tabular}

\footnotetext{
${ }^{1}$ Resultados expressos em base seca, média e DP.
} 
O teor de umidade da farinha do extrato da jabuticaba $(11,29 \%)$ apresentou-se dentro dos padrões da legislação vigente, no qual é estabelecido o limite de até 15\% de umidade para farinhas (BRASIL, 2005).

Em estudos com desenvolvimento de farinhas de jabuticaba, (Gurak et al., 2014) obtiveram valores similares de umidade ao da farinha do extrato da jabutiaba, sendo $15,2 \%$ no pó da casca e $11,5 \%$ no bagaço da jabuticaba. Já (Ascheri, Ascheri e Carvalho, 2006) obtiveram quase a metade do teor de umidade obtido nesta pesquisa, apresentando $7,3 \%$ na elaboração da farinha de bagaço de jabuticaba. Podendo ser explicado esse valor encontrado, por (Ascheri, Ascheri e Carvalho, 2006) elaborou a farinha ate chegar um valor de umidade baixo. Assim como outros estudos utilizando resíduos de frutos, como o caju, a goiaba, maracujá e a acerola, onde os teores de umidade variaram entre 3,3\% a 10,2\% (Aquino et al., 2010; Uchoa et al., 2008).

A baixa atividade de água obtida na fabricação de farinhas é considerada ideal para fins de prolongar a vida útil dos produtos, pois inibe o crescimento de micro-organismos patogênicos, além de impedirreações químicas e enzimáticas (Oliveira, Veloso e Teranortiz, 2009).

O teor de lipídios encontrado na farinha do resíduo da jabuticaba 0,6g/100g está próximo ao relatado por (Alves, 2011) em farinhas da casca de jabuticaba onde na secagem de $60^{\circ} \mathrm{C}$ foi de $0,56 \mathrm{~g} / 100 \mathrm{~g}$ de farinha. Ressaltando a baixa concentração de gorduras como um ponto positivo, fazendo com que este tenha menor quantidade de calorias e maior durabilidade, tendo menor oxidação lipídica.

O teor de proteínas encontrado na farinha do resíduo da jabuticaba $(12,13 \%)$ foi superior o descrito na literatura por (Marquetti, 2014) de 3,77g/100g na farinha da casca de jabuticaba, podendo ser explicado por causa da elaboração da farinha do extrato da jabuticaba com a polpa e casca. Já esta apresenta valor próximo ao descrito por (Ascheri, Ascheri e Carvalho, 2006)que relatou ter encontrado $11,00 \%$ na farinha de bagaço de jabuticaba fermentado.

O valor de fibras encontrado na farinha do extrato da jabuticaba foi de $9,35 \mathrm{~g} / 100 \mathrm{~g}$ apresentandose abaixo do relatado por (Alezandro et al., 2013) no fruto de jabuticaba de diferentes variedades com valores de $17,9 \mathrm{~g} / 100 \mathrm{~g}$ e 19,3g/100g, podendo ter sido subestimado pelo método de extração. Sendo superior ao encontrado por (Marquetti, 2014) na farinha da casca da jabuticaba de 5,81g/100g. (Dreher, 1995) relata que para um alimento ser considerado como uma boa fonte de fibra alimentar deve apresentar de 2 a $3 \%$ de fibra no alimento. No Brasil, por meio da Resolução ${ }^{\circ}$ 54 ,de 12 de novembro de 2012 da Secretaria Nacional de Vigilância Sanitária, se estabelece, que um alimento pode ser considerado fonte de fibra alimentar quando no produto pronto para consumo apresentar mínimo de $3 \mathrm{~g} / 100 \mathrm{~g}$ de fibras para alimentos sólidos e $3 \mathrm{~g} / 100$ $\mathrm{mL}$ de fibras para líquidos e alto conteúdo mínimo de $6 \mathrm{~g} / 100 \mathrm{~g}$ de fibras para alimentos sólidos. Sendo assim a farinha do extrato da jabuticaba pode ser considerado um alimento funcional alto conteúdo de fibras.

$\mathrm{O}$ valor de $\mathrm{pH}$ da farinha do extrato da jabuticaba $(3,5)$ se apresentou próximo aos resultados encontrados na literatura. Pesquisas com farinha da casca da manga e a farinha da casca do abacaxi, pó de resíduo de caju, de goiaba e de maracujá, relatam o pH variando de 4,4 a 4,6 (Costa et al., 2007; Mendes, 2013; Uchoa et al., 2008). Baixos valores de $\mathrm{pH}$ favorecem a estabilidade da antocianina (Inada et al., 2015).

A acidez apresentada na farinha do extrato da jabuticaba de $1,8 \%$, se aproxima ao descrito por (Azevêdo et al., 2008), que encontrou na farinha da casca da manga o teor de acidez de 1,9\%. Sendo o teor de acidez um importante parâmetro na avaliação do estado de conservação de um produto alimentício 
determinando a qualidade da farinha, pois a acidificação desempenha uma função inibidora do crescimento microbiano (Baruffadi e Oliveira, 1998; J A Ordonez, 2005). A legislação preconiza o limite de acidez para farinha de trigo comum de no máximo 3,0\% (BRASIL, 2005). Portanto a farinha do extrato da jabuticaba apresenta a acidez adequada ao preconizado pela legislação para farinhas.

A farinha do resíduo da jabuticaba apresentou os valores de cinzas 3,5g/100g. Estando próximo ao encontrado por (Ascheri, Ascheri e Carvalho, 2006)em estudo que avaliou a farinha de bagaço de jabuticaba, apresentando o valor de 3,4g/100g.

O percentual de açúcares totais da farinha do resíduo da jabuticaba obtido no presente estudo foi de $62,13 \%$, sendo este valor acima do encontrado na farinha do resíduo da acerola, 24,3\% (Aquino et al., 2010) e no encontrado na farinha da acerola (fruta), 43,2\% (SOARES et al., 2001). Este resultado pode ser explicado pela maior concentração de açúcares normalmente encontrados nas polpas do que nas cascas das frutas e por ser a acerola uma fruta mais ácida. As frutas, em sua maioria, possuem quantidades representativas de açúcares redutores (glicose e frutose), sendo feita essa determinação para se avaliar a potencialidade de fermentação do produto (Barros, Finger e Magalhães, 1996). Sendo o teor de carboidratos obtido na farinha do extrato da jabuticaba foi próximo ao encontrado por (Ascheri et al., 2006; Ascheri, Ascheri e Carvalho, 2006) na farinha da jabuticaba extrusada de $56,06 \%$ e (Ferreira et al., 2012) que relatou na casca de jabuticaba de 58,70\%. O valor elevado do teor de carboidratos demonstraa possibilidade desta farinha ser usada no enriquecimentode pães, biscoitos e outras receitas.

Em geral, a farinha do rextrato da jabuticaba encontra-se em conformidade com a composição físico-química de farinhas de resíduos publicadas na literatura (Alezandro et al., 2013; Borges et al., 2011; Rufino et al., 2011; Silva et al., 2014).

A Tabela 2 apresenta os valores de atividade e fenólicos totais da farinha do extrato da jabuticaba.

Tabela 2. Atividade Antioxidante (FRAP, ABTS) e Fenólicos Totais (Folin-Ciocalteu), da farinha do extrato da jabuticaba1.

\begin{tabular}{|c|c|c|c|c|}
\hline & $\begin{array}{c}\text { FRAP } \\
\left(\mu \mathrm{mol} \mathrm{Fe}{ }^{2+} / g\right)\end{array}$ & $\begin{array}{c}\text { ABTS } \\
(\mu \mathrm{mol} \text { Trolox } / \mathrm{g})\end{array}$ & $\begin{array}{c}\text { ABTS } \\
\left(\mu \mathrm{mol} \mathrm{AA}{ }^{2} / g\right)\end{array}$ & $\begin{array}{l}\text { Folin-Ciocalteu } \\
\left(\text { GAE }^{3} \mathrm{mg} / 100 \mathrm{~g}\right)\end{array}$ \\
\hline $\begin{array}{l}\text { Jabuticaba } \\
\text { (farinha do extrato) }\end{array}$ & $1419,9 \pm 135,9$ & $2505,5 \pm 696,2$ & $2548,6 \pm 690,6$ & $5410,3 \pm 123,9$ \\
\hline
\end{tabular}

1 Resultados expressos em base seca, média e DP por triplicata.

2 Equivalente de Ácido Ascórbico.

3 Equivalente de Ácido Gálico.

A atividade antioxidante foi avaliada por dois diferentes ensaios in vitro. A inexistência de um método universal e a influência de diferentes fatores na atividade antioxidante em matrizes alimentares complexas e heterogêneas, frequentemente pode não ser bem avaliada, usando apenas um protocolo de ensaio (Huang, Boxin e Prior, 2005). Portanto as duas metodologias escolhidas para avaliar a capacidade antioxidante dos alimentos, FRAP e ABTS, incluem os dois principais mecanismos de ação antioxidante, através da transferência de próton e elétron, respectivamente. Outra razão para esses dois ensaios terem sido escolhidos foi a possibilidade de comparação com dados existentes na literatura obtidos através dos mesmos.

A farinha do extrato da jabuticaba apresentou elevada atividade antioxidante, pelos dois métodos de análise (FRAP e ABTS). 
Recente pesquisa avaliou a composição química e atividade antioxidante da jabuticaba (Myrciaria jaboticaba) e, suas respectivas frações, sendo documentado na polpa 71,2 $\mu \mathrm{mol} \mathrm{Fe} \mathrm{Fe}^{2+} / \mathrm{g}$.E na casca da jabuticaba, 192,1 $\mu \mathrm{mol} \mathrm{Fe} \mathrm{F}^{2+} / \mathrm{g}$ (Inada et al., 2015).

Em outro estudo desenvolvido por (Marquetti, 2014) avaliando a atividade antioxidante na casca e na farinha de jabuticaba, por FRAP, obteve 242,7 e $168,1 \mu \mathrm{mol} \mathrm{Fe} \mathrm{Fe}^{2+} / \mathrm{g}$, respectivamente.

No presente estudo, a atividade antioxidante obtida na farinha do resíduo da jabuticaba (1419,8 $\mu \mathrm{molFe}{ }^{2+} / \mathrm{g}$ )apresentou-se de 7 a 20 vezes superior em comparação com a polpa e a casca da jabuticaba dos demais estudos mencionados (Inada et al., 2015; Marquetti, 2014). Podendo ser explicado pelo aumento da concentração de antioxidantes por ser a farinha do resíduo da jabuticaba elaborada somente com cascas e estas serem resíduos da elaboração do suco integral de jabuticaba.

(França, 2014)avaliando a atividade antioxidante em farinhas dos resíduos de frutas como a banana, a goiaba e a manga obtiveram resultados inferiores ao da farinha de extrato da jabuticaba, sendo na ordem de 24,7; 22,4; 69,9 $\mathrm{mmolFe}^{2+} / \mathrm{g}$, respectivamente. Os valores dessas farinhas de outras frutas se apresentaram inferiores, até mesmo a valores de Atividade antioxidante em suco de fruta integral,como apresentadono suco de uva integral, 32,7 $\mu \mathrm{mol} \mathrm{Fe} \mathrm{Fe}^{2+}$ g (Dias e Menegon, 2012).

$\mathrm{Na}$ análise da farinha do extrato da jabuticaba, pelo método ABTS, o resultado expresso foi de 2505,5 $\mu$ molTrolox /g e 2548,6 $\mu \mathrm{mol}$ AA/g. Nosso resultado se apresenta superior a outros estudos que analisaram a jabuticaba pelo método de ABTS, tendo o fruto da jabuticaba apresentado 138,0 $\mu$ molTrolox /g, a casca da jabuticaba, de 638,6 a 723.8 $\mu$ molTrolox /ge a farinha de jabuticaba 468,5 $\mu$ molTrolox /g (Marquetti, 2014; Rufino et al., 2010; SILVA et al., 2010).
Espécies de açaí foram descritas (Kang et al., 2012) e, apresentaram valores de atividade antioxidanteque variaram de 985,9 a 1792,3 uM TE $\mathrm{g}$ 1. Tais valores de Atividade antioxidante encontrados no açaí se equivalem proporcionalmente aos valores da casca da jabuticaba, talvez justificado pela similaridade entre esses frutos. Valores menos expressivos foram detectados na casca de uva da variedade Santa Isabel de 89,2 $\mu \mathrm{Mol}$ TEAC/g e no bagaço seco de uva variedade Pinot Gris de 463,4 $\mu \mathrm{Mol}$ TEAC/g (Cataneo et al., 2008; Soares et al., 2008).

Em estudo de liofilizado da farinha de jabuticaba obteve 9458 uM TEAC g-1(Leite-Legatti et al., 2012). Este elevado valor de Atividade Antioxidante na farinha liofilizada de jabuticaba pode ser devido ao processo de liofilização, concentrar e conservar de maneira mais eficaz os compostos antioxidantes, do que a desidratação por estufa ventilada, como utilizada em no presente estudo para a elaboração da farinha do extrato da jabuticaba.

Avaliaram pelo método de ABTS, farinhas de resíduos de acerola, de graviola e de tangerina, sendo os resultados para atividade antioxidante, respectivamente, 247,2; 310,7 e 78,8 $\mu \mathrm{mol}$ de Trolox /g de amostra(SENA et al., 2014).

Em farinhas alimentícias, reconhecidas como boas fontes de antioxidantes, sendo estas: gergelim, linhaça, aveia, açafrão, girassol, milho, trigo, soja, os valores da atividade antioxidante variaram de: 43 a 1230; sendo maior teor encontrado na farinha de açafrão (Herculano, 2014). Tendo a farinha de açafrão a que apresentou a maior atividade antioxidante, mesmo assim a metade do valor verificado no presente estudo, na farinha do extrato de jabuticaba.

Além da elevada atividade antioxidante encontrada na farinha do extrato da jabuticaba, a quantificação de fenólicos totais demonstrou o teor 5410mg GAE/100g de amostra, sendo este 
teorpróximo ao encontrado por Leite-Legatti et al.,(2012) na farinha liofilizada da jabuticaba (5563mg GAE/100g) e, superior ao encontrado por (Gurak et al., 2014), que obteve no bagaço e no pó da casca da jabuticaba (4339mg GAE/100g e 3024mg GAE/100g, respectivamente).

Em estudo avaliando o resíduo de seriguela, outra fruta exótica brasileira, o teor de fenólicos totais foi de 4229mg GAE/100g, valor bem próximo ao encontrado na farinha do resíduo da jabuticaba (Silva e Melo, 2013).

(Perin e Schott, 2011) em seu estudo com o resíduo de uva para elaboração de biscoitos tipo cooks, obteve o total de fenólicos de 49,94mg GAE/100g, enquanto que (Pereira et al., 2013) descreveram 88,38mg GAE/100g na farinha do resíduo de acerola,teores estes inferiores ao encontrado na farinha do extrato da jabuticaba.

Os compostos fenólicos constituem os antioxidantes mais representativos em frutos e vegetais, sendo o teor de fenólicos totais utilizado como importante indicador da capacidade antioxidante total de alimentos e bebidas, portanto este pode ser usado para rastreio preliminar para que o produto possa considerando ser como fonte natural de antioxidantes (Viuda-Martos et al., 2011).

Como descrito pela literatura que resíduos de suco de frutas possuem quantidades expressivas de compostos fenólicos, por causa do modo de extração, incluindo o aquecimento e prensagem do fruto para a produção do suco, o que afeta significativamente a composição fenólica do bagaço. Portanto, o aquecimento durante a produção de suco de jabuticaba aumenta o teor de polifenóis totais, porque o tratamento térmico pode produzir mudanças na estabilidade, devido ao rompimento da parede celular da planta (Gurak et al., 2014). Este fato pode explicar o motivo pelo qual no presente estudo, da farinha do extrato da jabuticaba elaborada a partir do suco integral de jabuticaba por aquecimento e desidratação, apresentou maior teor de fenólicos totais quando comparado com a literatura.

O elevado teor de antioxidantes e fenólicos totais encontrados na farinha do extrato da jabuticaba se respalda na evidência de existir uma ampla variedade de compostos fenólicos, tais como antocianinas, se concentrarem majoritariamente nas cascas (Bicudo, Ribani e Beta, 2014; Borges et al., 2011; Rufino et al., 2010; Wu et al., 2012).

Em relação a benefícios a saúde, pesquisas experimentais recentes tem demonstrado que a administração diária de extrato de jabuticaba é eficaz para controlar o estresse oxidativo e a hiperlipidemia, pois, os polifenóis possuem forte atividade inibidora da lipase pancreática. Neste sentido, a utilização da farinha do extrato de jabuticaba pode ser uma alternativa de elevada potencial antioxidante (Alezandro et al., 2013). E beneficia a saúde.

\section{CONCLUSÃO}

A caracterização físico-química da farinha do extrato da jabuticaba permitiu comprovar a adequação dentro dos padrões estabelecidos pela legislação.

A farinha obtida a partir do extrato da jabuticaba apresentou um expressivo teor de compostos antioxidantese de compostos fenólicos totais, podendo ser viável a sua utilização para o desenvolvimento de subprodutos de maior valor agregado, promovendo assim, uma forma de comercialização e consumo saudável e sustentável de frutos nativos do Brasil. E utilização deste para auxiliar no tratamento de doenças crônicas não-transmissíveis.

\section{REFERÊNCIAS BIBLIOGRÁFICAS}

ALEZANDRO, M. R.; DUBÉ, P.; DESJARDINS, Y.; LAJOLO, F. M.; GENOVESE, M. I. 
Comparative study of chemical and phenolic compositions of two species of jaboticaba: Myrciaria jaboticaba (Vell.) Berg and Myrciaria cauliflora (Mart.) O. Berg. Food Research International, v. 54, n. 1, p. 468-477, 2013.

ALVES, A. P. DE C. Casca de jabuticaba (Plinia jaboticaba (Vell.) Berg): Processo de secagem e uso como aditivo em iogurte. [s.l.] Universidade Federal de Lavras, 2011.

AQUINO, A. C. M. DE S.; MÓES, R. S.; LEÃO, K. M. M.; FIGUEIREDO, A. V. D.; CASTRO, A. A. Avaliação físico-química e aceitação sensorial de biscoitos tipo cookies elaborados com farinha de resíduos de acerola Physical-chemical and sensory characteristics of cookies formulated with acerola (Malpighia emarginata D.C.) residues flour. Rev Inst Adolfo Lutz, v. 69, n. 3, p. 379-86, 2010.

ASCHERI, D. P. R.; ANDRADE, C. T.; CARVALHO, C. W. P.; ASCHERI, J. L. R. Efeito da extrusão sobre a adsorção de água de farinhas mistas prégelatinizadas de arroz e bagaço de jabuticaba. Ciencia e Tecnologia de Alimentos, v. 26, n. 2, p. 325-335, 2006.

ASCHERI, D. P. R.; ASCHERI, J. L. R.; CARVALHO, C. W. P. Caracterização da farinha de bagaço de Jabuticaba e propriedades funcionais dos extrusados. Ciencia e Tecnologia de Alimentos, v. 26, n. 4, p. 897-905, 2006.

ASSOCIAÇÃO BRASILEIRA DE FRANCHISING. Pesquisa Food Service ABF. Disponível em: <https://www.abf.com.br/pesquisa-foodservice-abf-2015>.

AZEVÊDO, L. CAVALCANTI DE; AZOUBEL, P. M.; SLIVA, I. RAFAELA ALVES; ARAÚJO, A. JÚLIA DE B.; OLIVEIRA, SILVANA BELEM DE. CARACTERIZAÇÃO FÍSICOQUÍMICA DA FARINHA DA CASCA DE MANGA cv. TOMMY ATKINS. XXI Congresso Brasileiro de Ciência e Tecnologia de Alimentos. UFV., v. 2, p. 2-5, 2008.

BARROS, R. S.; FINGER, F. L.; MAGALHÃES, M. $\mathrm{M}$. Changes in non-structural carbohydrates in developing fruit of Myrciaria jaboticaba. Scientia Horticulturae, v. 66, n. 3-4, p. 209215, 1996.

BARUFFADI, R.; OLIVEIRA, M. N. Fundamentos de tecnologia de alimentos. Atheneu Ed ed. São Paulo: [s.n.].

BENZIE, I. F. F.; STRAIN, J. J. Ferric reducing/antioxidant power assay: Direct measure of total antioxidant activity of biological fluids and modified version for simultaneous measurement of total antioxidant power and ascorbic acid concentration. Methods in Enzymology, v. 299, n. 1995, p. 15-27, 1999.

BICUDO, M. O. P.; RIBANI, R. H.; BETA, T. Anthocyanins, Phenolic Acids and Antioxidant Properties of Juçara Fruits (Euterpe edulis M.) Along the On-tree Ripening Process. Plant Foods for Human Nutrition, v. 69, n. 2, p. 142-147, 2014.

BORGES, G. D. S. C.; VIEIRA, F. G. K.; CRISTIANE COPETTI; GONZAGA, L. V.; ZAMBIAZI, R. C.; FILHO, J. M.; FET'T, R. Chemical characterization, bioactive compounds, and antioxidant capacity of jussara (Euterpe edulis) fruit from the Atlantic Forest in southern Brazil. Food Research International, v. 44, n. 7, p. 2128-2133, 2011.

BRAGA A C D, LIMA M S, AZEVEDO L C, R. E. C. Caracterização e obtenção de farinha do resíduo gerado no processo industrial de clarificação do suco de acerola. Rev Semiárido De Visu, v. 1, p. 126-133, 2011.

BRASIL. Resolução de Diretoria Colegiada - RDC No 263, de 22 de setembro de 2005Ministério da Saúde Agência Nacional de Vigilância Sanitária, , 2005

CATANEO, C. B.; CALIARI, V.; VALDEMIRO GONZAGA, L.; MARTA KUSKOSKI, E.; FET'T, R. Semina: Ciências Agrárias Atividade antioxidante e conteúdo fenólico do resíduo agroindustrial da produção de vinho Antioxidant activity and phenolic content of agricultural byproducts from wine production. Semina: Ciências Agrárias, v. 29, n. 1, p. 93-101, 2008.

COSTA, J. M. C. DA; FELIPE, É. M. DE F.; GERALDO ARRAES MAIA, I.; MONTENEGRO BRASIL, F.; HERNANDEZ, F. H. Comparação dos parâmetros físico-químicos e químicos de pós alimentícios obtidos de resíduos de abacaxi. Revista Ciência Agronômica, v. 38, n. 2, p. 228-232, 2007. 
DANNER, M. A.; CITADIN, I.; SASSO, S. A. Z.; TOMAZONI, J. C. Diagnóstico ecogeográfico da ocorrência de jabuticabeiras nativas no sudoeste do Paraná. Revista Brasileira de Fruticultura, v. 32, n. 3, p. 746-753, 2010.

DERAL. Anuário Brasileiro De Fruticultura 2017. editora gazeta, 2017.

DIAS, SIMONE DE PAULA; MENEGON, RENATO FARINA. Comparação do teor de fenólicos totais e da ação antioxidante e sucos industrializados de uva e de vinhos tinto. Revista Univap, p. 68-76, 2012.

DONADIO, L. C. Jabuticaba (Myrciaria jaboticaba (Vell. Berg). Jaboticabal: FUNEP, p. 55, 2000.

DREHER, M L. Food industry perspective: functional properties and food uses of dietary fiber. Minnesota: Eagan Press: [s.n.].

FERREIRA, A. E.; MARIA, M.; LAGES, B.; MARIA, P.; THÉ, P. PRODUÇÃO, CARACTERIZAÇÃO E UTILIZAÇÃO DA FARINHA DE CASCA DE JABUTICABA EM BISCOITOS TIPO COOKIE. Alim. Nutr, v. 23, n. 4, p. 603-607, 2012.

FRANÇA, F. DE A. CARACTERIZAÇÃO NUTRICIONAL E AVALIAÇÃO DO POTENCIAL ANTIOXIDANTE DE FARINHAS OBTIDAS DE RESÍDUOS DE FRUTAS. [s.l.] Universidade Estadual do Sudoeste da Bahia (UESB), 2014.

GURAK, P. D.; BONA, G. S. DE; TESSARO, I. C.; MARCZAK, L. D. F. Jaboticaba pomace powder obtained as a co-product of juice extraction: A comparative study of powder obtained from peel and whole fruit. Food Research International, v. 62, p. 786-792, 2014.

HALVORSEN, B. L.; CARLSEN, M. H.; PHILLIPS, K. M.; BØHN, S. K.; HOLTE, K.; JACOBS, D. R.; BLOMHOFF, R. Content of redox-active compounds (ie, antioxidants) in foods consumed in the United States. American Journal of Clinical Nutrition, v. 84, n. 1, p. 95-135, 2006.

HERCULANO, K. L. Capacidade antioxidante de farinhas alimentícias. [s.l: s.n.].

HUANG, D.; BOXIN, O. U.; PRIOR, R. L. The chemistry behind antioxidant capacity assays. Journal of Agricultural and Food Chemistry, v. 53, n. 6, p. 1841-1856, 2005.

IAL. Normas Analíticas do Instituto Adolfo Lutz: métodos químicos e físicos para Análise de alimentos. Métodos físicos-quimicos para análise de Alimentos, p. 1020, 2008.

INADA, K. O. P.; OLIVEIRA, A. A.; REVORÊEO, T. B.; MARTINS, A. B. N.; LACERDA, E. C. Q.; FREIRE, A. S.; BRAZ, B. F.; SANTELLI, R. E.; TORRES, A. G.; PERRONE, D.; MONTEIRO, M. C. Screening of the chemical composition and occurring antioxidants in jabuticaba (Myrciaria jaboticaba) and jussara (Euterpe edulis) fruits and their fractions. Journal of Functional Foods, v. 17, p. 422433, 2015

INSTITUTO DE ECONOMIA AGRÍCOLA. Dinâmica da Fruticultura Paulista: aspectos mercadológicos 2013-2017. Análises e Indicadores do Agronegócio , v. 14, 2019.

J A ORDONEZ. Tecnologia de alimentos Alimentos de origem animal. Artmed ed. Porto Alegre: [s.n.].

KANG, J.; THAKALI, K. M.; XIE, C.; KONDO, M.; TONG, Y.; OU, B.; JENSEN, G.; MEDINA, M. B.; SCHAUSS, A. G.; WU, X. Bioactivities of açaí (Euterpe precatoria Mart.) fruit pulp, superior antioxidant and anti-inflammatory properties to Euterpe oleracea Mart. Food Chemistry, v. 133, n. 3, p. 671-677, 2012.

LEITE-LEGATTI, A. V.; BATISTA, A. G.; DRAGANO, N. R. V.; MARQUES, A. C.; MALTA, L. G.; RICCIO, M. F.; EBERLIN, M. N.; MACHADO, A. R. T.; CARVALHOSILVA, L. B. DE; RUIZ, A. L. T. G.; CARVALHO, J. E. DE; PASTORE, G. M.; MARÓSTICA, M. R. Jaboticaba peel: Antioxidant compounds, antiproliferative and antimutagenic activities. Food Research International, v. 49, n. 1, p. 596-603, 2012.

MARQUETTI, C. Obtenção e caracterização de farinha de casca de jaboticaba (Plinia cauliflora) para adição em biscoito tipo cookie. [s.l.] UNIVERSIDADE TECNOLÓGICA FEDERAL DO PARANÁ MESTRADO, 2014.

MENDES, B. A. B. Obtenção, caracterização e 
aplicação de farinha das cascas de abacaxi e de manga. [s.1.] Universidade Estadual do Sudoeste da Bahia, 2013.

NILE, S. H.; PARK, S. W. Edible berries: Bioactive components and their effect on human health. Nutrition, v. 30, n. 2, p. 134-144, 2014.

OLIVEIRA, LUANA TAMIRY; VELOSO, J. CRISTINA RIBEIRO; TERANORTIZ, G. PATRICIA. Caracterização físico-química da farinha de semente e casca de uva.SEMANA DE CIÊNCIA E TECNOLOGIA DO IFMG campus Bambuí II JORNADA CIENTIFICAMinas GeraisIn: SEMANA DE CIÊNCIA E TECNOLOGIA DO IFMG campus Bambuí II JORNADA CIENTIFICA, , 2009 .

OLIVEIRA, A. L. DE; BRUNINI, M. A.; SALANDINI, C. A. R.; BAZZO, F. R. Caracterização tecnológica de jabuticabas "Sabará" provenientes de diferentes regiões de cultivo. Revista Brasileira de Fruticultura, v. 25, n. 3, p. 397-400, 2003.

PEREIRA, C. T. M.; SILVA, C. R. DOS P.; LIMA, A. DE; PEREIRA, D. M.; COSTA, C. DO N.; NETO, A. A. C. Obtenção, caracterização físicoquímica e avaliação da capacidade antioxidante in vitro da farinha de resíduo de acerola( Malpighia glabra L.). Acta Tecnológica, v. 8, n. 2, p. 5056, 2013.

PERIN, E. C.; SCHOTT, I. B. Utilização de farinha extraída de resíduos de uva na elaboração de biscoito tipo cookie. p. 62, 2011.

RE, R.; PELLEGRINI, N.; PROTEGGENTE, A.; PANNALA, A.; YANG, M.; RICE-EVANS, C. ANTIOXIDANT ACTIVITY APPLYING AN IMPROVED ABTS RADICAL CATION DECOLORIZATION ASSAY. Free Radic Biol Med, v. 26, p. 1231-1237, 1999.

RUFINO, M. D. S. M.; ALVES, R. E.; BRITO, E. S. DE; SILVEIRA, M. R. S. DA; MOURA, C. F. H. Quality for fresh consumption and processing of some non-traditional tropical fruits from Brazil. Fruits, v. 64, n. 6, p. 361-370, 2009.

RUFINO, M. DO S. M.; ALVES, R. E.; BRITO, E. S. DE; PÉREZ-JIMÉNEZ, J.; SAURACALIXTO, F.; MANCINI-FILHO, J. Bioactive compounds and antioxidant capacities of 18 non-traditional tropical fruits from Brazil. Food
Chemistry, v. 121, n. 4, p. 996-1002, 2010.

RUFINO, M. S. M.; ALVES, R. E.; FERNANDES, F. A. N.; BRITO, E. S. Free radical scavenging behavior of ten exotic tropical fruits extracts. Food Research International, v. 44, n. 7, p. 2072-2075, 2011.

SANTOS, D. T.; VEGGI, P. C.; MEIRELES, M. A. . Extraction of antioxidant compounds from Jabuticaba (Myrciaria cauliflora) skins: yield, composition and economical evaluation. J. Food Eng, v. 101, p. 23-31, 2010.

SENA, D. N. ; SOUSA, M. M. A. ; SOUSA, P. H. M. ; ALMEIDA, M. M. B. Estudo do Potencial Antioxidante em Amostras de Farinha de ResíXX congress Brasileiro de engenharia químicaFlorianópolis., 2014.

SILVA, G. J. F. DA; CONSTANT, P. B. L; FIGUEIREDO, R. W. DE; MOURA, S. M. FORMULAÇÃO E ESTABILIDADE DE CORANTES DE ANTOCIANINAS EXTRAÍDAS DAS CASCAS DE JABUTICABA (MYRCIARIA ssp.). Alim. Nutr, v. 21, p. 429-436, 2010.

SILVA, J. K. DA. Aqueous extract of jaboticaba peel (Myrciaria jaboticaba): antioxidant, antiinflammatory effects and impact on intestinal microbiota in healthy rats and with TNBS-induced colitis. [s.1.] UNIVERSIDADE ESTADUAL DE CAMPINAS, 2017.

SILVA, N. A. DA; RODRIGUES, E; MERCADANTE, A. Z.; ROSSO, V. V. DE. Phenolic compounds and carotenoids from four fruits native from the Brazilian Atlantic forest. Journal of Agricultural and Food Chemistry, v. 62, n. 22, p. 5072-5084, 2014.

SILVA, T. M. DA; MELO, E. DE A. RESÍDUO DE CIRIGUELA (SPONDIAS PURPUREA L): TEOR DE FITOQUIMICOS E POTENCIAL ANTIOXIDANTErecifeXIII JORNADA DE ENSINO, PESQUISA E EXTENSÃO - JEPEX 2013 - UFRPE:, , 2013.

SINGLETON, V. L.; ORTHOFER, R.; LAMUELARAVENTÓS, R. M. Analysis of Total Phenols and Other Oxidation Substrates and Antioxidants by Means of Folin-Ciocalteu Resgent. [s.l: s.n.]. v. 299 
SOARES, E. C.; OLIVEIRA, G. S. F. DE; MAIA, G. A.; MONTEIRO, J. C. S.; SILVA JR., A.; S. FILHO, M. DE S. DE. DESIDRATAÇÃO DA POLPA DE ACEROLA (Malpighia emarginata D.C.) PELO PROCESSO "FOAM-MAT". Ciência e Tecnologia de Alimentos, v. 21, n. 2, p. 164-170, 2001.

SOARES, M.; WELTER, L.; KUSKOSKI, E. M.; GONZAGA, L.; FET'T, R.; DE. COMPOSTOS FENÓLICOS E ATIVIDADE ANTIOXIDANTE DA CASCA DE uva. Rev. Bras. Frutic., Jaboticabal -, v. 30, n. 1, p. 5964, 2008.

SZAJDEK, A.; BOROWSKA, E. J. Bioactive compounds and health-promoting properties of Berry fruits: A review. Plant Foods for Human Nutrition, v. 63, n. 4, p. 147-156, 2008.

TORRES, T.; FARAH, A. Coffee, maté, açaí and beans are the main contributors to the antioxidant capacity of Brazilian's diet. European Journal of Nutrition, v. 56, n. 4, p. 1523-1533, 2017.

UCHOA, A. M. A.; COSTA, J. M. C. DA; MAIA, G. A.; SILVA, E. M. C.; CARVALHO, A. DE F. F. U.; MEIRA, T. R. Parâmetros Físico-Químicos , Teor de Fibra Bruta e Alimentar Physicochemical Parameters and Crude and Dieary Fiber Content of Edible Powders From Tropical Fruit Residues. Segurança Alimentar e Nutricional, v. 15, n. 2, p. 58-65, 2008.
VIUDA-MARTOS, M.; RUIZ-NAVAJAS, Y.; FERNÁNDEZ-LÓPEZ, J.; PÉREZÁLVAREZ, J. A. Spices as functional foods. Critical Reviews in Food Science and Nutrition, v. 51, n. 1, p. 13-28, 2011.

VIZZOT'TO, M. V; PEREIRA, M C. Metodologia Científica: Otimização do Processo de Extração de Compostos Fenólicos Antioxidantes de Mirtilo (Vaccinium ashei Reade)Embrapa ClimaTemperadopelotas ,RsBoletim de Pesquisa e Desenvolvimento, , 2009 .

WU, S. B.; DASTMALCHI, K.; LONG, C.; KENNELLY, E. J. Metabolite profiling of jaboticaba (Myrciaria cauliflora) and other darkcolored fruit juices. Journal of Agricultural and Food Chemistry, v. 60, n. 30, p. 7513-7525, 2012.

WU, S. B.; LONG, C.; KENNELLY, E. J. Phytochemistry and health benefits of jaboticaba, an emerging fruit crop from Brazil. Food Research International, v. 54, n. 1, p. 148-159, 2013.

YANG, Q.; WANG, H. C.; LIU, Y.; GAO, C.; SUN, L.; TAO, L. Resveratrol Cardioprotection Against Myocardial Ischemia/Reperfusion Injury Involves Upregulation of Adiponectin Levels and Multimerization in Type 2 Diabetic Mice. Journal of Cardiovascular Pharmacology, v. 68, n. 4, p. 304-312, 2016. 